\title{
Human impacts on polycyclic aromatic hydrocarbon distribution in Chinese intertidal
} \section{zones}

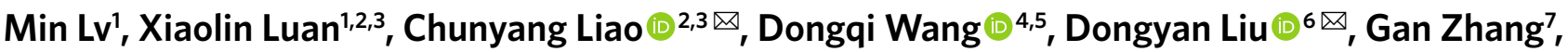 \\ Guibin Jiang ${ }^{2,3}$ and Lingxin Chen $\mathbb{1 D}^{1,3,8} \bowtie$
}

The intertidal zone-a transitional boundary between terrestrial and marine environments-has important ecological functions, and receives polycyclic aromatic hydrocarbons (PAHs) from human activities, but how and to what extent anthropogenic factors influence the distribution of PAHs in this important niche remain largely unknown. Here we measured the distribution of United States Environmental Protection Agency priority PAHs in samples of intertidal sediments from across more than 4,500 km of China's coastline. The total PAH concentrations ranged from 2.3 to $1,031.7 \mathrm{ng} \mathrm{g}^{-1} \mathrm{sediment}^{(\mathrm{dry}}$ weight) and all PAHs showed positive correlations with total organic carbon (TOC). TOC-normalized high-molecular-weight (HMW) PAH concentrations, but not TOC-normalized low-molecular-weight (LMW) PAHs, were positively correlated with TOC. Moreover, population size and economic development influenced TOC-normalized HMW PAH concentrations, whereas urbanization had a major influence on TOC-normalized LMW PAHs. Human activities also indirectly influenced TOC-normalized PAH concentrations by affecting TOC. In sum, our investigation provides continental-scale evidence that human activities have key and differential effects on the distribution and deposition of PAHs in intertidal sediments, and shows that pollution status and profile of PAHs can be used to index regional industrialization and urbanization status.

$\mathrm{P}$ AHs are hydrophobic organic pollutants containing two or more fused benzene rings. They have been identified as priority pollutants by the United States Environmental Protection Agency ${ }^{1}$. PAHs can be produced by incomplete combustion of carbonaceous materials and release of petroleum products ${ }^{2}$; release of PAHs into the environment is thus viewed as unavoidable. The semivolatile and persistent properties of PAHs enhance their dissemination in the environment and widespread occurrences of PAHs in air, surface water, soils, sediments and biota have been documented ${ }^{3-8}$. The intertidal zone, as the intersection of terrestrial and marine environments, has important hydrological and ecological functions ${ }^{9,10}$. The typical ecosystem of the intertidal zone is fragile and easily affected by human activities ${ }^{11,12}$. Industrialization and urbanization have resulted in the discharge of large amounts of contaminants into intertidal zones during the past few decades. PAHs are highly likely to be deposited in intertidal sediments due to their high hydrophobicity.

National and global atmospheric PAH emissions can be estimated from fuel consumption data ${ }^{13-15}$. Burning of biomass and domestic coal are the dominant sources of PAH emissions in China, where emissions are higher during winter ${ }^{14,15}$. Many factors influence the distribution of PAHs in intertidal sediments, including surface runoff, atmospheric deposition, ocean source (that is, PAHs can be sourced from the ocean, such as the release of petroleum products from maritime traffic) and water-sediment interactions.
However, little is known about how these factors influence the distribution of PAHs in the intertidal environment. As anthropogenic pollutants with differences in distribution and composition depending on the type and intensity of the source, PAHs are potential geochemical indicators for assessing human influence within a specific environmental medium ${ }^{16,17}$. Recent studies have shown seasonal variations in concentrations of PAHs in surface waters in the East China Sea and Yellow Sea ${ }^{18}$, and in intertidal sediments at most sampling sites ${ }^{19}$. Moreover, sediment properties such as TOC and clay content have been reported to correlate with PAH concentrations in intertidal zones ${ }^{20}$ but not in soils ${ }^{21,22}$. Therefore, it is critical to explore how and to what extent various anthropogenic and/ or geological factors affect the distribution of PAHs in the intertidal environment. This will not only help to identify the effects of human activities on the distribution of PAHs, but will also support the development of prediction models for deposition of PAHs in sediments from intertidal zones and elsewhere.

A key element of predicting concentrations of pollutants from human activities is an understanding of how and to what extent anthropogenic factors influence the distribution of the pollutants in the intertidal environment, which remains largely unknown. In this study, we used samples from more than 150 sites in 14 intertidal zones along the coastline of China (Fig. 1 and Supplementary Table 1). Sediments were sampled during both wet and dry seasons. The large variations in climate (temperature and rainfall) and

\footnotetext{
${ }^{1}$ CAS Key Laboratory of Coastal Environmental Processes and Ecological Remediation, Yantai Institute of Coastal Zone Research, Chinese Academy of Sciences, Yantai, China. ${ }^{2}$ State Key Laboratory of Environmental Chemistry and Ecotoxicology, Research Center for Eco-Environmental Sciences, Chinese Academy of Sciences, Beijing, China. ${ }^{3}$ University of Chinese Academy of Sciences, Beijing, China. ${ }^{4}$ School of Geographic Sciences, East China Normal University, Shanghai, China. ${ }^{5}$ Key Laboratory of Geographic Information Science of the Ministry of Education, East China Normal University, Shanghai, China. ${ }^{6}$ State Key Laboratory of Estuarine and Coastal Research, Institute of Eco-Chongming, East China Normal University, Shanghai, China. ${ }^{7}$ State Key Laboratory of Organic Geochemistry, Guangzhou Institute of Geochemistry, Chinese Academy of Sciences, Guangzhou, China. ${ }^{8}$ Center for Ocean Mega-Science, Chinese Academy of Sciences, Qingdao, China. ${ }^{凶}$ e-mail: cyliao@rcees.ac.cn; dyliu@sklec.ecnu.edu.cn; Ixchen@yic.ac.cn
} 

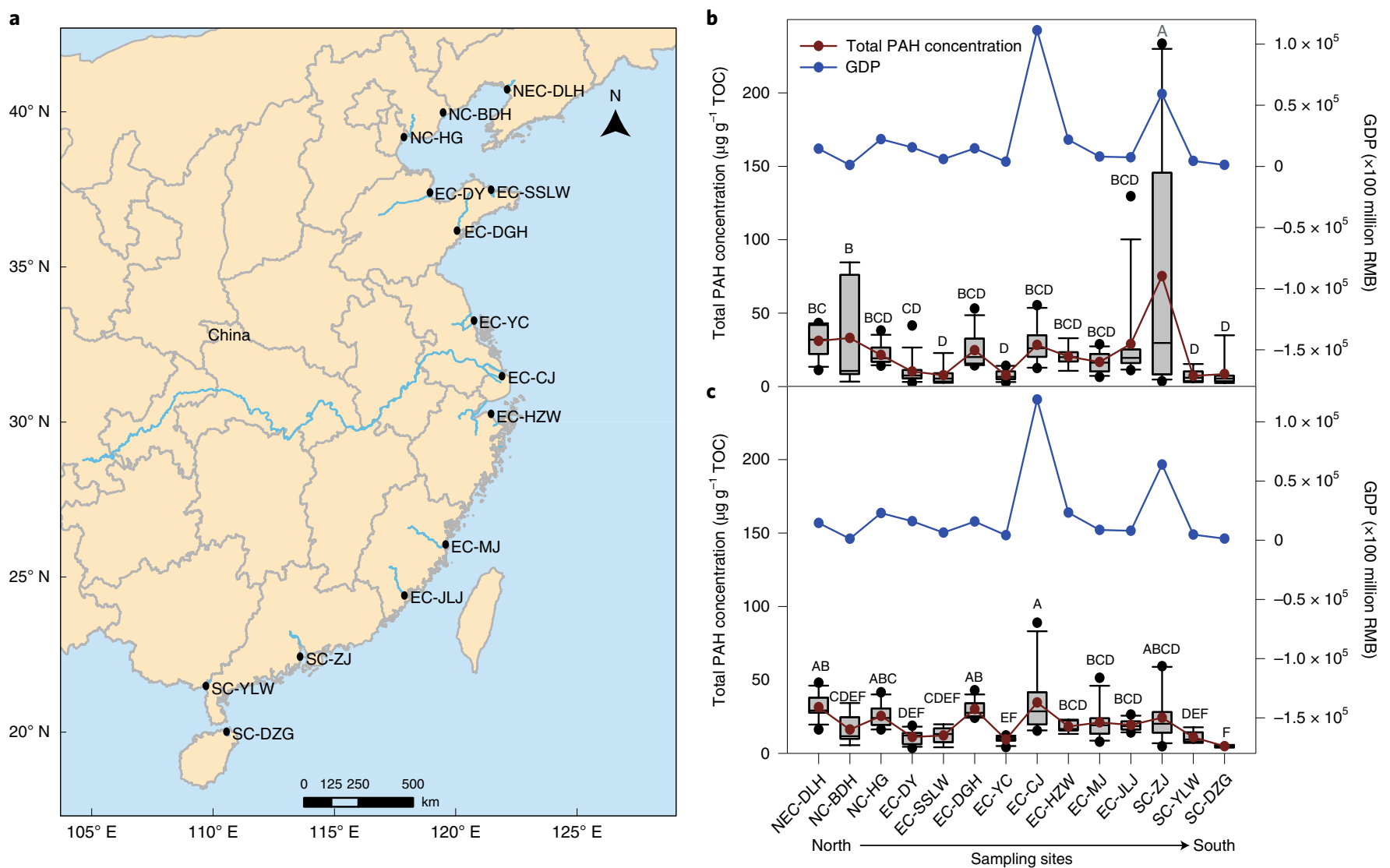

Fig. 1 Sampling sites and PAH concentrations. a, Sampling sites spanning over all coastal provinces and municipalities in China (see Supplementary Table 1 for detailed information of the abbreviations of sampling sites). b,c, Total PAH concentrations in samples of intertidal sediments from different sites and the corresponding gross domestic product (GDP) in dry (b) and wet (c) seasons. The data for the map shown in a are from http://www.resdc.cn/. In box plots (b,c), the centre line represents the median $\mathrm{PAH}$ concentration; red dots represent the mean; box edges indicate the 25th and 75th percentiles; whiskers extend to data points not considered outliers; outliers are plotted using the ' $\bullet$ ' symbol. Different letters (A, B, C, D, E, F) represent differences are significant at $P<0.05$.

anthropogenic factors (socio-economic parameters) provided an opportunity to investigate how diverse factors influence distribution of PAHs in intertidal zones (Supplementary Table 2). Structure equation model (SEM) analysis was used to further resolve the direct and indirect effects of the various factors (anthropogenic factors, TOC and climate factors) on PAH profiles in intertidal sediments. This study fills an important knowledge gap by identifying the distribution patterns of PAHs in the intertidal environment on a continental scale and exploring the associations of PAHs in sediments with human activities. Overall, our results show spatial variations in the distribution and composition of PAHs in the intertidal zones, which reflect levels of urbanization and industrialization in the studied regions.

\section{Results}

This section summarizes the PAH distribution patterns in the intertidal sediments, the key factors that influence them, and considerations about the use of diagnostic ratios for source attribution.

PAH distribution patterns in the intertidal sediments. The total amount of 15 PAH congeners $\left(\Sigma_{15}\right.$ PAHs) in the sediments of the 14 intertidal zones from north to south China ranged from 2.3 to $1,031.7 \mathrm{ng} \mathrm{g}^{-1}$ sediment (Supplementary Fig. 1 and Supplementary Table 3) and from 2.4 to $233.5 \mu \mathrm{gg}^{-1}$ TOC (Fig. 1). Divergent spatial variations were found for PAH concentrations and TOC-normalized PAHs concentrations, with sediments collected from EC-JLJ and
SC-ZJ containing the highest non-normalized and TOC-normalized $\mathrm{PAH}$ concentrations, respectively. The highly hydrophopic nature of PAHs and the high TOC in sediment from EC-JLJ resulted in high PAH concentrations at this site. When normalized by TOC content, the PAH concentration was largely dependent on the input of PAHs. This was verified by the strong positive correlation of TOC with $\mathrm{PAH}$ concentrations (Supplementary Fig. 2), and the agreement of TOC-normalized PAH concentrations with the regional economic parameter gross domestic product $(r=0.42, P<0.01$; Fig. 1$)$. Notably, TOC-normalized PAH concentrations showed divergent correlations with the TOC (Fig. 2 and Supplementary Table 4): TOC-normalized concentrations of 3-ring (LMW) PAHs decreased with increasing TOC $(r=-0.18, P<0.01)$, whereas those of 4 - to 6-ring (HMW) PAHs increased with TOC $(r=0.41, P<0.01)$.

No obvious temporal variations were found in non-normalized and TOC-normalized $\Sigma_{15} \mathrm{PAHs}$ in the intertidal sediments (Fig. 1 and Supplementary Fig. 1), with the exception of sediments from NC-HG, EC-SSLW and EC-YC, in which samples from the wet season contained higher concentrations of $\Sigma_{15} \mathrm{PAHs}$ and TOC-normalized 3-ring PAHs $(P<0.05)$. As TOC-normalized concentrations of 3-ring PAHs correlated with rainfall on a continental scale $(r=0.25, P<0.01$; Supplementary Table 4$)$, the above results indicate that although $\mathrm{PAH}$ emissions in China are markedly higher in winter than in summer ${ }^{14}$, other factors, such as higher rainfall in the wet season, could cause large inputs of PAHs into the intertidal zones through atmospheric deposition. 

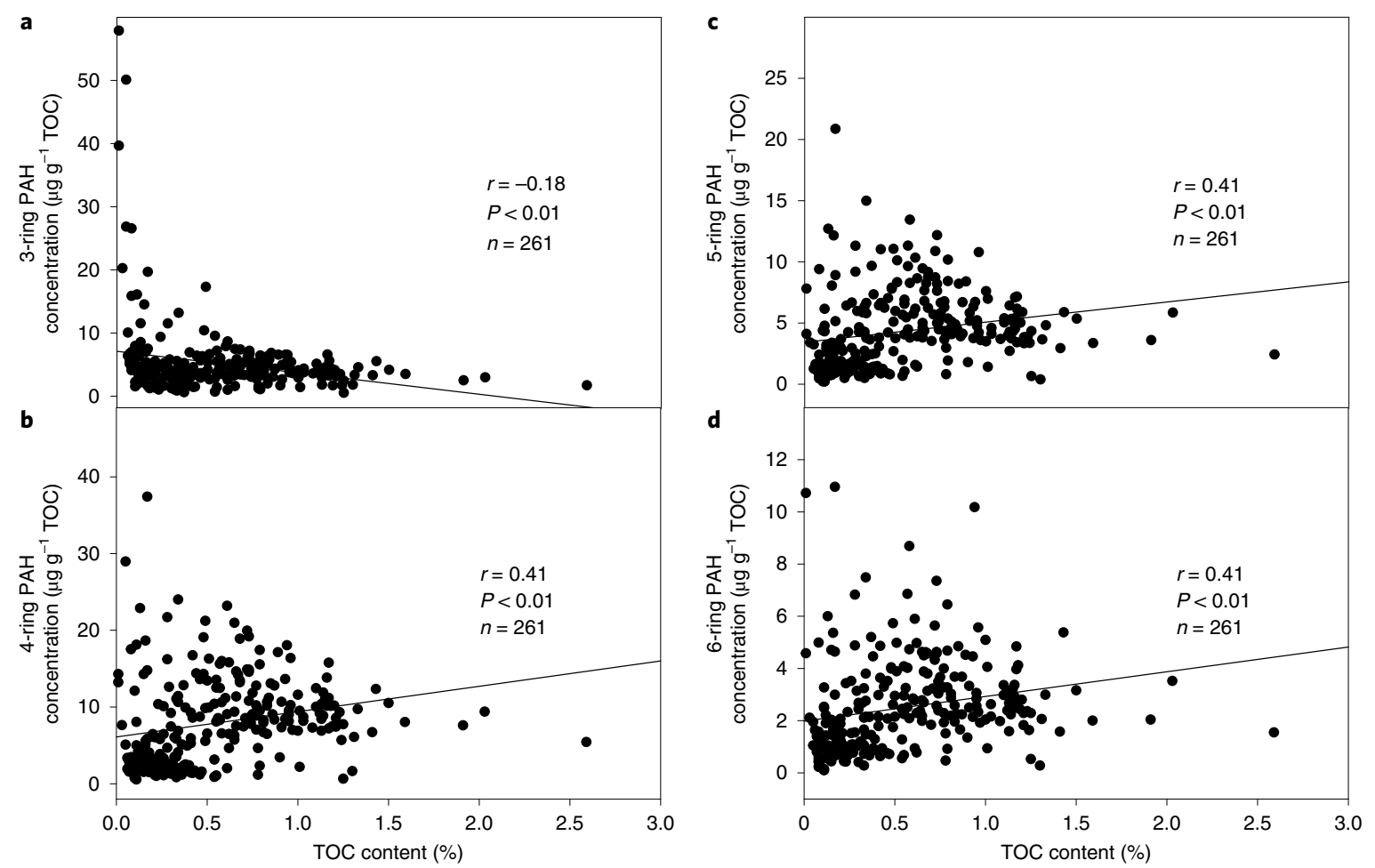

Fig. 2 | Relationships between PAH concentrations and TOC. a-d, Spearman correlations between TOC-normalized concentrations of 3-ring (a), 4-ring (b), 5-ring (c) and 6-ring PAHs (d) and TOC in intertidal sediments.

Spatiotemporal variations were found in the compositions of PAH compounds in the sediments (Supplementary Fig. 3 and Supplementary Tables 3 and 5), with higher ratios of 3-ring PAHs observed in sediments from NC-BDH, EC-SSLW, SC-YLW and EC-YC, and the highest ratios of 4- to 6-ring PAHs found in samples from NEC-DLH, NC-HG, EC-DGH, EC-CJ, EC-HZW and EC-JLJ. In most intertidal zones, although the mean ratios of 4- to 6-ring PAHs were somewhat higher in the dry season than in the wet season, this trend was not significant $(P>0.05)$. This was further validated by principal component analysis (PCA) (Supplementary Fig. 4). No obvious separated clusters were observed among the different seasons (Supplementary Fig. 4a) and different tide levels (Supplementary Fig. 4b), but spatial variations in $\mathrm{PAH}$ concentrations levels were found among different intertidal zones; EC-JLJ, NEC-DLH, NC-HG, EC-DGH, EC-HZW and EC-CJ clustered together and were separated from the other intertidal zones (Supplementary Fig. 4c). 3-ring PAHs, including phenanthrene (Phe), fluorene (Flu), acenaphthene (Ace), acenaphthylene (Acy) and anthracene (Ant) drove the separation from the clustered intertidal zones. Of note, 4-ring PAHs including fluoranthene (Fla), pyrene (Pyr), benzo[a]anthracene $(\mathrm{BaA})$ and chrysene $(\mathrm{Chr})$ drove the separation of EC-JLJ, NEC-DLH and NC-HG from the other intertidal zones, whereas 5- and 6-ring PAHs drove the separation of EC-HZW and EC-CJ from the other zones. Comparison of concentrations and compositions of PAHs with those reported previously is shown in detail in Supplementary Table 3 and discussed in Supplementary Discussion. Notably, the intertidal flat surface sediments of the Yangtze estuary in $2000^{23}$ and Jiulong River estuary in $1999^{24}$ exhibited different PAH compositions compared with the corresponding EC-CJ and EC-JLJ in the present study, with 2- to 3-ring PAHs and 5-ring PAHs predominant, respectively, indicating that the sources of PAHs in these areas have probably changed.
PAH sources and considerations regarding diagnostic ratio. Although molecular diagnostic ratios including $\mathrm{Fla} /(\mathrm{Fla}+\mathrm{Pyr})$, $\mathrm{BaA} /(\mathrm{BaA}+\mathrm{Chr}), \mathrm{Ant} /(\mathrm{Phe}+\mathrm{Ant})$ and Ind/(Ind + BghiP) have been widely used for identifying and assessing the origin of $\mathrm{PAHs}{ }^{25,26}$, it has been argued that these ratios could be altered due to differences in the fate of PAHs in the complex environment, resulting in substantial changes in diagnostic ratios between source emission and environmental sample ${ }^{27,28}$. As higher values of these diagnostic ratios indicate larger contributions from pyrogenic sources, including combustion of coal and biomass, the present study provides us an opportunity to assess the diagnostic ratios on a continental scale by evaluating their relationships with coal and oil consumption. Although the ratios (mean \pm s.d.) of $\mathrm{Fla} /(\mathrm{Fla}+\mathrm{Pyr})=0.55 \pm 0.07$, $\mathrm{BaA} /(\mathrm{BaA}+\mathrm{Chr})=0.36 \pm 0.07, \mathrm{Ant} /(\mathrm{Phe}+\mathrm{Ant})=0.13 \pm 0.05$ and Ind $/($ Ind + BghiP $)=0.49 \pm 0.11$ across all sites suggest that the PAHs originated mainly from combustion of coal, biomass and liquid fuel (Supplementary Fig. 5 and Supplementary Table 6), significant and negative correlations were observed between Fla/(Fla + Pyr) and Ant/(Phe + Ant), coal consumption, oil consumption, respectively $(P<0.05)$ (Supplementary Fig. 6 and Supplementary Table 7). The negative correlations of Fla/(Fla + Pyr) with coal consumption, the wide range of diagnostic ratios in the same intertidal zone (Supplementary Fig. 6 and Supplementary Table 6) and the inconsistency of the trend in diagnostic ratios (Fig. 3 and Supplementary Fig. 6) indicate that diagnostic ratios may have been altered substantially in the sediment, giving rise to concern about the use of diagnostic ratios to identify sources. Of note, Ant/(Phe+Ant) was positively correlated with both coal consumption and oil consumption $(P<0.01)$, in particular for sediments from sites in northern China, where Ant/(Phe + Ant) showed good agreement with energy consumption (Fig. 3 and Supplementary Table 7). These results suggest that Ant/(Phe+Ant) in intertidal sediments reliably reflect the consumption of coal and oil in north China, which is consistent 


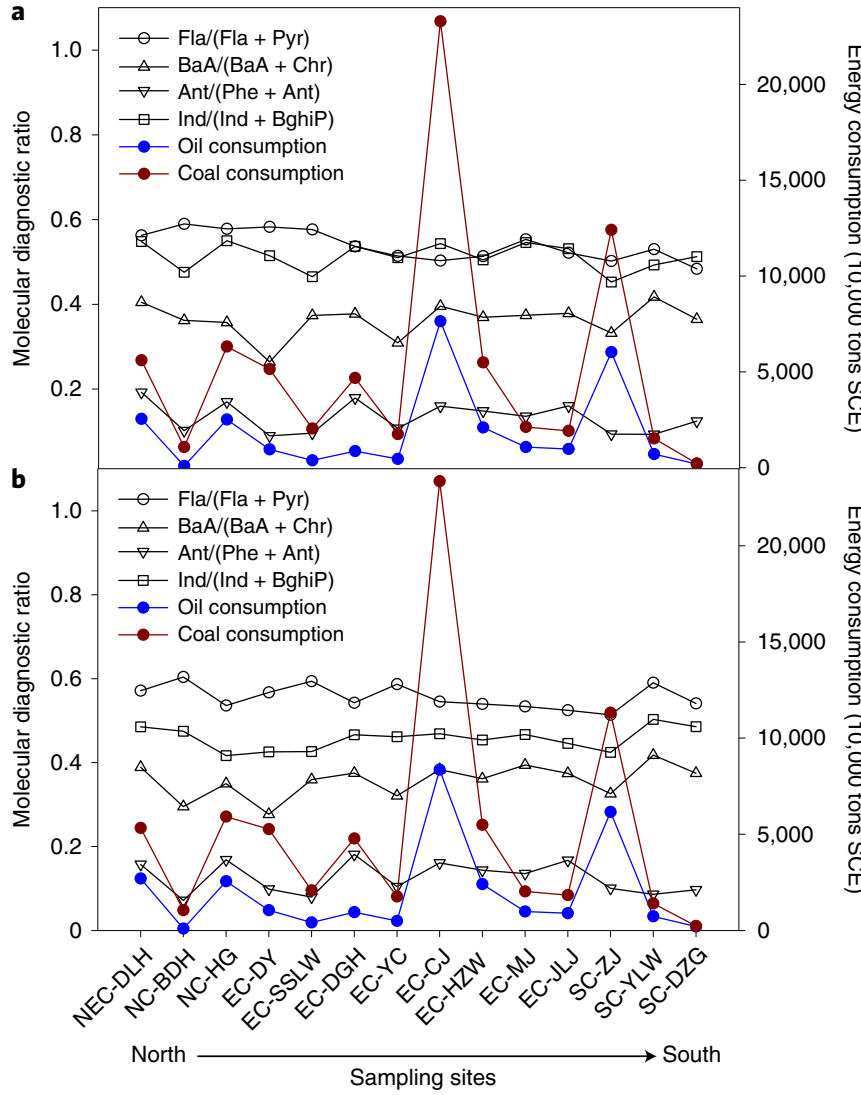

Fig. 3 | Distribution of molecular diagnostic ratios and energy consumption. a,b, Mean values of molecular diagnostic ratios and energy consumption in intertidal sediments sampled during dry (a) and wet (b) seasons. SCE, standard coal equivalent; Ind, indeno[1,2,3-cd]pyrene; BghiP, benzo[g,h,i]perylene.

with the findings of Zhang et al. ${ }^{27}$ showing that among the PAHs, the receptor-to-source ratios of Ant and Phe are most similar in sediment $^{27}$. Nevertheless, the significant and positive correlation with TOC in some intertidal zones $(P<0.05$; Supplementary Table 8$)$ and on a continental level $(r=0.65, P<0.01$; Supplementary Table 7) suggest a high probability that Ant/(Phe + Ant) was altered from the ratio at the source. Since Ant and Phe have similar octanol-water partition coefficient $\left(\log K_{\mathrm{ow}}\right)$ values, the positive correlation with TOC was probably due to the shorter biodegradation half-life of Phe (63.8d) compared with Ant (107d) (https://comptox.epa.gov/dashboard), and high TOC usually indicates abundant microorganisms, which could result in faster elimination of Phe and thus higher values of Ant/(Phe + Ant). Similarly, the negative correlation between Fla/(Fla + Pyr) and TOC in most intertidal zones (Supplementary Table 8) and on a continental level $(r=-0.29$, $P<0.01$; Supplementary Table 7) could be explained by the shorter biodegradation half-life of Fla (163d) than Pyr (255d) (https:// comptox.epa.gov/dashboard). Thus, the above results provide evidence that the diagnostic ratios were probably altered during transport and sedimentation, particularly in sediments with higher TOC.

PCA coupled with multilinear regression analysis (PCA-MLR) was further used to investigate the source of PAHs. On the basis of results from previous studies ${ }^{15,29-32}$, PCA-MLR of our results indicated PAH contributions of $40.2 \%$ from combustion of coal, biomass and fossil fuels, $33.8 \%$ from combustion of coal and biomass and $26.0 \%$ from mixed sources of petrogenic PAHs and petroleum combustion (Supplementary Tables 9-11; see Supplementary Information for detailed discussion). These results are consistent with statistical data of energy consumption in China showing that consumption of coal and oil account for $38.9-88.5 \%$ and $7.0-34.4 \%$ of the total energy consumption in the studied area, respectively (Supplementary Table 2), and that biomass such as straw and firewood are widely used in rural areas ${ }^{14}$.

Factors influencing PAHs patterns in intertidal zones. The strong correlation between PAHs and sediment physicochemical properties (TOC and content of clay, silt and sand) on a continental scale, and in particular the stronger positive correlation between PAHs and TOC in sediment from intertidal zones (Supplementary Fig. 2 and Supplementary Table 12) are understandable since TOC and fine grain sizes provide adsorption surfaces for PAHs. Interestingly, despite the unknown pollution sources and complex hydraulic conditions in the intertidal zones at different sampling sites, TOC showed positive correlations with PAHs for about half of the intertidal zones (Supplementary Table 8), indicating the possibility of redistribution of PAHs at these sites. However, previous studies on PAHs in Chinese and British soils found no correlations between soil organic matter and total or heavy PAH concentrations $s^{21,22}$. These results suggested that diverse factors influence the distribution of PAHs in inland soils and sediment in intertidal zones. Intertidal zones are at the intersection of marine and terrestrial environments, and receive PAHs from multiple sources including riverine input, surface runoff, atmospheric deposition and ocean currents, and deposit PAHs after substantial hydrological processes. Under complex conditions such as those described above, sediment properties, particularly TOC, determine its capacity to adsorb and deposit PAHs. Therefore, given the large variations in TOC $(0.01-2.59 \%)$ in our sediment samples, TOC-normalized PAH concentrations were considered.

To investigate the factors influencing the spatiotemporal distribution patterns of TOC-normalized PAHs in intertidal zones, SEMs encompassing 34 anthropogenic and climatic parameters (temperature and rainfall) were used. The 34 anthropogenic parameters were grouped into six categories using PCA; the categories included population size and economic development, industrial structure and air quality, urbanization, waste gas emissions, per capita oil consumption and use of traditional energy sources (coal and oil) (Supplementary Table 13). The SEMs produced divergent results for TOC-normalized HMW and LMW PAHs (Fig. 4). Population size and economic development $(\lambda=0.35, P<0.001)$, industrial structure and air quality $(\lambda=0.10, P<0.05)$, urbanization $(\lambda=0.18, P<0.001)$, waste gas emissions $(\lambda=0.27, P<0.001)$, per capita oil consumption $(\lambda=0.25, P<0.001)$, traditional energy source ratio $(\lambda=0.29$, $P<0.001)$ and TOC $(\lambda=0.21, P<0.001)$ had positive effects on TOC-normalized HMW PAHs, whereas traditional energy source ratio $(\lambda=0.12, P<0.05)$, urbanization $(\lambda=-0.40, P<0.001)$ and TOC $(\lambda=-0.17, P<0.001)$ had direct effects on TOC-normalized LMW PAHs. Since urbanization can positively influence TOC, it also indirectly influenced HMW PAHs $(\lambda=0.03)$ and LMW PAHs $(\lambda=-0.03)$. Notably, despite the influence of population size and economic development on TOC-normalized HMW PAH concentration, they had no effects on TOC-normalized LMW PAHs. Urbanization was the dominant factor for TOC-normalized LMW PAHs. Climate factors had no direct effects on TOC-normalized HMW or LMW PAHs, but showed indirect effects via waste gas emissions or traditional energy source ratio, which was reflected in negative correlations between these two factors and climate factors (temperature and rainfall) $(P<0.05$; Supplementary Table 14). Waste gas emissions and traditional energy source ratio were higher in north China and during the dry season (in 2014), compared with south China and the wet season (in 2015) (Supplementary Table 2).

While parameters related to maritime traffic had the highest loadings for the population size and economic development (Supplementary Table 13), the dominant and positive effect of population size and economic development on TOC-normalized 

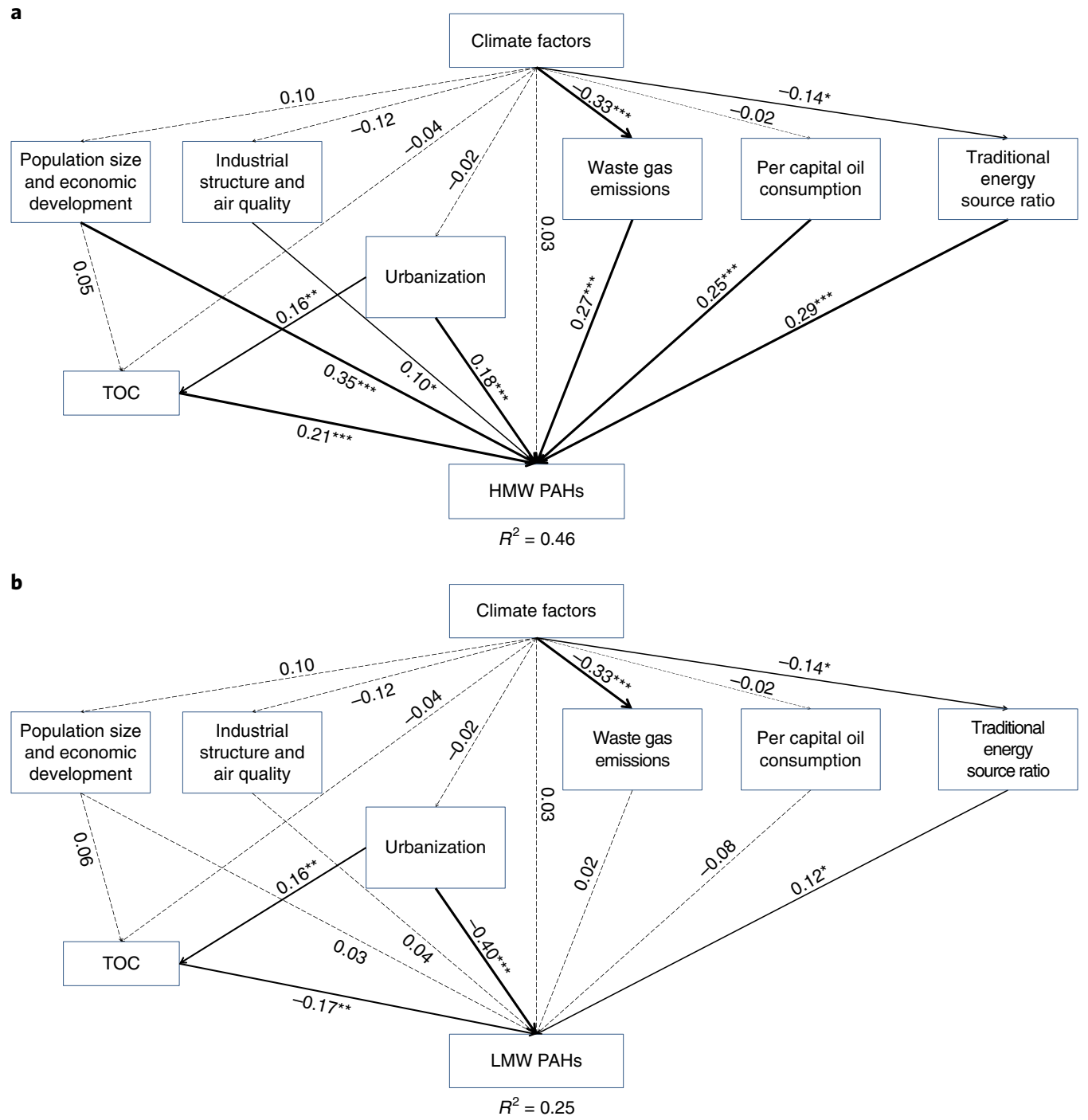

Fig. 4 | Structural equation models showing the direct and indirect effects of TOC, climate factors and anthropogenic factors on TOC-normalized PAH concentrations. a,b, Numbers adjacent to the arrows are path coefficients and indicative of the effect size of the relationship. Width of solid lines indicates the strength of the path coefficients. Dashed lines indicate nonsignificant relationships. ${ }^{\star} P<0.05,{ }^{\star \star} P<0.01$ and ${ }^{\star \star \star} P<0.001$. $R^{2}$ represents the proportion of variance explained by the relations in the path model. The hypothetical models satisfactorily fitted our data with $\chi^{2}=17.8, P=0.40$, d.f. $=17, \mathrm{GFI}=0.99$, $\mathrm{AIC}=74$ and $\mathrm{RMSEA}=0.01(\mathbf{a})$ and $\chi^{2}=2.2, P=1.000$, d.f. $=16, \mathrm{GFI}=1.00, \mathrm{AIC}=60$ and $\mathrm{RMSEA}=0.00(\mathbf{b})$.

HMW PAH concentrations implied that increased PAH emissions from both terrestrial sources and maritime traffic made important contributions to the deposition of HMW PAHs in intertidal zones with large population sizes and economic development. Notably, air quality parameters were clustered with industrial structure rather than waste gas emissions (Supplementary Table 13), indicating the key role of industrial structure in determining air quality; a high ratio of heavy industry results in increases in parameters describing poor air quality, such as concentrations of particulates less than $10 \mu \mathrm{m}\left(\mathrm{PM}_{10}\right)$ or $2.5 \mu \mathrm{m}\left(\mathrm{PM}_{2.5}\right)$ in size, and increased deposition of HMW PAHs in intertidal sediments. These results indicate that optimization of industry structure may be an efficient way to improve air quality and reduce emissions of HMW PAHs. The effects of coal and oil use on TOC-normalized HMW and LMW PAHs indicates that decreasing the consumption of these fuels can reduce $\mathrm{PAH}$ pollution.

The divergent effects of TOC on TOC-normalized HMW and LMW PAHs indicates that higher TOC results in increased deposition of HMW PAHs and decreased deposition of LMW
PAHs. This is reflected by the positive correlation of TOC with TOC-normalized HMW PAHs $(P<0.01)$ and the negative correlation with TOC-normalized LMW PAHs $(P<0.01)$ (Supplementary Table 4). LMW PAHs have a lower octanol-water partition coefficient and higher volatility compared with HMW PAHs. The positive correlations of rainfall with TOC-normalized LMW PAHs (Supplementary Table 4) further suggest that wet deposition through rainfall is an important source of LMW PAHs. Urbanization was positively correlated with all PAHs (Supplementary Table 15), but higher urbanization rate resulted in higher TOC-normalized HMW PAH concentrations and lower TOC-normalized LMW PAH concentrations (Fig. 4). This could be explained by the higher input of TOC and optimized techniques to reduce the emission of LMW PAHs in areas with higher urbanization rate ${ }^{13}$. The negative effects on LMW PAHs were verified by the negative correlation between TOC-normalized LMW PAHs and urbanization (Supplementary Table 4).

The parameters describing air quality and waste gas emissions were based on regional data, whereas the other anthropogenic 
parameters were localized to the river basins. The effects of these anthropogenic factors on PAH profiles suggest that rivers are an important source of PAH deposition in the intertidal zones, particularly for HMW PAHs. Notably, although we have observed seasonal variations of PAHs in surface water ${ }^{18}$ and intertidal sediment ${ }^{19}$ at most sampling sites in previous studies, we did not observe seasonal variations in PAHs in this study (Fig. 1 and Supplementary Fig. 1) and the direct effect of climate factors on PAH profiles was not significant $(P>0.05$; Fig. 4$)$. These results indicate that while PAH emissions were higher in winter ${ }^{14}$, improvements in industry structure, energy consumption ratio and wet deposition through rainfall in the wet season (in 2015) may have obscured any seasonal variations.

\section{Discussion}

The intertidal zone, a space that has frequently been overlooked by land and ocean scientists, is a very important transitional zone between land and ocean, with a high intensity of human activity. This study attempts to disentangle the factors influencing $\mathrm{PAH}$ profiles in intertidal sediments on a continental scale. Deposition of PAHs in intertidal sediments, resulting from economic development and traditional energy source consumption, merit increased attention. We have shown that economic activity, energy consumption, the ratio of heavy industry, urbanization rate, waste gas emissions, per capita oil consumption and traditional energy source ratio directly and indirectly result in increases in TOC-normalized HMW PAHs (Fig. 4). Increased urbanization is accompanied by increased TOC content in intertidal sediment across the continent, corroborating the enhanced deposition of HMW PAHs in highly urbanized regions. By contrast, TOC-normalized LMW PAH concentrations were higher in areas with less urbanization and lower TOC content, despite the positive effect of traditional energy consumption ratio, providing clear evidence for the divergent distribution patterns of TOC-normalized HMW and LMW PAH concentrations. Furthermore, despite higher emission of PAHs into the atmosphere during the dry season ${ }^{14}$, increases in economic activity, energy consumption, urbanization rate and per capita oil consumption led to higher PAHs input during the wet season (in 2015), which obscured the temporal variations in PAHs. The number of intertidal zones selected for this study was limited due to the muddy environment and difficulty of sampling, and future extension of the spatial distribution of sampling sites will enable more comprehensive insights. Nevertheless, the 14 intertidal zones examined here include sites in all 11 Chinese coastal provinces and municipalities across more than $4,500 \mathrm{~km}$ of coastline, and the large variations in terms of climate (temperature and rainfall), geographical location and socio-economic development status enabled anthropogenic parameters to be linked to spatial variations in PAHs (Fig. 1 and Supplementary Table 2). Our findings provide information about how diverse factors influence the distribution of PAHs in intertidal zones. Collectively, this study provides insights into the roles of sediment properties, human activities and climate factors in the spatiotemporal distribution of PAHs in intertidal sediments on a continental scale, and advances our understanding of the mechanisms that drive PAH distribution patterns. We have shown that the distribution of PAHs - and particularly that of HMW PAHscan reflect the regional development status, with implications for prediction and risk assessment in relation to PAH accumulation in other intertidal regions.

\section{Methods}

Sampling in intertidal zones. Surface sediments (depth $0-5 \mathrm{~cm}$ ) were taken from 14 intertidal zones in the dry season (September 2014 to February 2015) and the wet season (July to September 2015), across all 11 coastal provinces and municipalities in China (Fig. la and Supplementary Table 1). For each intertidal zone, three to five transects perpendicular to the coastline were selected, and low, middle and high tides were generally set for each transect. A total of 315 samples were obtained for dry and wet seasons. Sediment samples were transported on dry ice, freeze-dried and stored at $-20^{\circ} \mathrm{C}$ until further analysis. Extraction and analysis of PAHs is described in detail in the Supplementary Methods.

Quality assurance and quality control. All PAHs were analysed in selected reaction monitoring (SRM) mode, using the two highest characteristic precursor ion-product ion transition pairs (Supplementary Table 16). Surrogate standards were added to all samples before extraction to monitor method performance. A procedural blank, a spiked blank, a matrix spike and a duplicate were run for each batch of samples to assess potential sample contamination and reliability of the data. Calibration was done by analysing a standard solution to ensure that the deviation of the measured concentrations was within $20 \%$ of the initial calibration values. The respective spike recoveries for PAHs in sediment samples are shown in Supplementary Table 16. The method detection limits for the analysed PAHs were between 0.01 and $1.0 \mathrm{ngg}^{-1}$ (Supplementary Table 16). Because of the potential evaporative losses and high levels detected in blank samples during the chemical analysis, naphthalene was excluded from further considerations. Concentrations of PAHs were not corrected against the recovery of surrogates. Non-normalized and TOC-normalized PAH concentrations were reported as $\mathrm{ng} \mathrm{g}^{-1}$ dry weight and $\mu \mathrm{gg}^{-1} \mathrm{TOC}$, respectively. Total concentrations of 15 PAH congeners were expressed as $\Sigma_{15}$ PAHs.

Environmental factors and anthropogenic factors. TOC was measured with an elemental analyser (Micro Club Elementar). Particle-size distribution of the sediment was analysed using a laser diffraction particle-size analyser (Mastersizer 3000). Thirty-four anthropogenic parameters and climatic parameters (temperature and rainfall) were collected or estimated from governmental statistical yearbooks (Supplementary Table 2).

Statistical analysis. One-way analysis of variance (ANOVA), two-tailed Spearman correlations and PCA-MLR were performed using SPSS 18.0. Since 261 samples analysed for TOC were in the appropriate range, statistical analyses were generally based on these 261 samples.

SEM was used to quantify the direct and indirect effects of the multiple drivers on TOC-normalized PAHs. Dimension-reduction analysis was commonly used to avoid redundancy among highly correlated variables and refine the main dimensions of variations for further modelling ${ }^{33,34}$. Factor analysis was used to reveal the relationships underlying the multiple variables in this study. Six main components were extracted from 34 anthropogenic parameters with eigenvalues of $10.8,9.5,3.3,2.8,2.8$ and 1.5 ; they accounted for $31.7 \%, 28.0 \%$, $9.6 \%, 8.3 \%, 8.1 \%$ and $4.4 \%$ of the variation, respectively. On the basis of the loadings of the parameters (Supplementary Table 13), the six main components were population size and economic development, industrial structure and air quality, urbanization, waste gas emissions, per capita oil consumption and traditional energy source ratio. Two components with eigenvalues above unity were extracted from the 15 TOC-normalized PAHs, accounting for $83.5 \%$ of the total variation (Supplementary Table 17). The first component was HMW PAHs, with high loadings $>0.8$ for 4 - to 6-ring PAHs and 0.66 for Ant. The second component was represented by 3-ring PAHs, and was identified as LMW PAHs. We then proposed a priori conceptual SEMs depicting the direct and indirect effects of TOC, climate factors, the population size and economic development, industrial structure and air quality, urbanization, waste gas emissions, per capita oil consumption and traditional energy source ratio on profiles of HMW and LMW PAHs (Supplementary Fig. 7). Specifically, we hypothesized that: (1) TOC could directly affect the PAH profiles; (2) since the anthropogenic parameters were on an annual basis, the SEM was developed with potential flows of causality from climate factors to all anthropogenic factors to indicate their spatial and annual variations, considering the higher temperature and rainfall in south China and the wet season (Supplementary Table 2). Moreover, climate factors could directly influence the patterns of PAHs through deposition. They could also indirectly affect $\mathrm{PAH}$ profiles by altering TOC and affecting anthropogenic parameters; and (3) for anthropogenic factors, population size and economic development and urbanization could directly and indirectly influence PAH profiles by causing emission of more PAHs into the environment, altering TOC content and affecting $\mathrm{PAH}$ profiles, while industrial structure and air quality, waste gas emissions, per capita oil consumption and traditional energy source ratio could directly influence PAH profiles. Bivariate correlation and factor analysis were performed using SPSS 18.0 and the SEM construction using maximum-likelihood estimation was performed with AMOS 21 (SPSS).

The overall goodness of fit for SEM was generally tested using multiple criteria: $\chi^{2}$-test (the model has a good fit when $0 \leq \chi^{2} \leq 2$ and $0.05<P \leq 1.00$ ), the root mean square error of approximation $(0 \leq \mathrm{RMSEA} \leq 0.05)$, and a high goodness-of-fit index (GFI $>0.90)$. With a good fit, the influence of one variable on another was calculated by summing all direct and indirect pathways between them and the effect is analogous to the bivariate correlation coefficient between the two variables ${ }^{35}$.

Reporting Summary. Further information on research design is available in the Nature Research Reporting Summary linked to this article. 


\section{Data availability}

The data supporting the findings of this study are summarized in the Supplementary Information, and any other data analysed in the current study are available from the corresponding author upon request.

Received: 6 May 2019; Accepted: 1 June 2020;

Published online: 29 June 2020

\section{References}

1. Keith, L. \& Telliard, W. Priority pollutants: I-a perspective view. Environ. Sci. Technol. 13, 416-423 (1979).

2. Ravindra, K., Sokhi, R. \& Van Grieken, R. Atmospheric polycyclic aromatic hydrocarbons: source attribution, emission factors and regulation. Atmos. Environ. 42, 2895-2921 (2008).

3. Lang, C., Tao, S., Liu, W. X., Zhang, Y. X. \& Simonich, S. Atmospheric transport and outflow of polycyclic aromatic hydrocarbons from China. Environ. Sci. Technol. 42, 5196-5201 (2008).

4. Han, D. M. \& Currell, M. J. Persistent organic pollutants in China's surface water systems. Sci. Total Environ. 580, 602-625 (2017).

5. Hamid, N. et al. A review on the abundance, distribution and eco-biological risks of PAHs in the key environmental matrices of South Asia. Rev. Environ. Contam. Toxicol. 240, 1-30 (2017).

6. Balcioglu, E. B. Potential effects of polycyclic aromatic hydrocarbons (PAHs) in marine foods on human health: a critical review. Toxin Rev. 35, 98-105 (2016).

7. Zhang, P. \& Chen, Y. G. Polycyclic aromatic hydrocarbons contamination in surface soil of China: a review. Sci. Total Environ. 605, 1011-1020 (2017).

8. Lunde, G. \& Bjorseth, A. Polycyclic aromatic-hydrocarbons in long-range transported aerosols. Nature 268, 518-519 (1977).

9. Barbier, E. B. et al. The value of estuarine and coastal ecosystem services. Ecol. Monogr. 81, 169-193 (2011).

10. Wamsley, T. V., Cialone, M. A., Smith, J. M., Atkinson, J. H. \& Rosati, J. D. The potential of wetlands in reducing storm surge. Ocean Eng. 37, 59-68 (2010).

11. Doney, S. C. The growing human footprint on coastal and open-ocean biogeochemistry. Science 328, 1512-1516 (2010).

12. Lotze, H. K. et al. Depletion, degradation, and recovery potential of estuaries and coastal seas. Science 312, 1806-1809 (2006).

13. Shen, H. Z. et al. Global atmospheric emissions of polycyclic aromatic hydrocarbons from 1960 to 2008 and future predictions. Environ. Sci. Technol. 47, 6415-6424 (2013).

14. Zhang, Y. X. \& Tao, S. Seasonal variation of polycyclic aromatic hydrocarbons (PAHs) emissions in China. Environ. Pollut. 156, 657-663 (2008).

15. Xu, S. S., Liu, W. X. \& Tao, S. Emission of polycyclic aromatic hydrocarbons in China. Environ. Sci. Technol. 40, 702-708 (2006).

16. Mai, B. X. et al. Distribution of polycyclic aromatic hydrocarbons in the coastal region off Macao, China: assessment of input sources and transport pathways using compositional analysis. Environ. Sci. Technol. 37, 4855-4863 (2003)

17. Liu, L. Y., Wang, J. Z., Wei, G. L., Guan, Y. F. \& Zeng, E. Y. Polycyclic aromatic hydrocarbons (PAHs) in continental shelf sediment of China: implications for anthropogenic influences on coastal marine environment. Environ. Pollut. 167, 155-162 (2012).

18. Ya, M. L. et al. Seasonal variation of terrigenous polycyclic aromatic hydrocarbons along the marginal seas of China: input, phase partitioning, and ocean-current transport. Environ. Sci. Technol. 51, 9072-9079 (2017).

19. Li, X. F. et al. Polycyclic aromatic hydrocarbons and black carbon in intertidal sediments of China coastal zones: concentration, ecological risk, source and their relationship. Sci. Total Environ. 566, 1387-1397 (2016).

20. Keshavarzifard, M., Moore, F., Keshavarzi, B. \& Sharifi, R. Distribution, source apportionment and health risk assessment of polycyclic aromatic hydrocarbons (PAHs) in intertidal sediment of Asaluyeh, Persian Gulf. Environ. Geochem. Health 40, 721-735 (2018).

21. Heywood, E. et al. Factors influencing the national distribution of polycyclic aromatic hydrocarbons and polychlorinated biphenyls in British soils. Environ. Sci. Technol. 40, 7629-7635 (2006).
22. Syed, J. H. et al. Polycyclic aromatic hydrocarbons (PAHs) in Chinese forest soils: profile composition, spatial variations and source apportionment. Sci. Rep. 7, 2692 (2017).

23. Liu, M. et al. Distribution and sources of polycyclic aromatic hydrocarbons in intertidal flat surface sediments from the Yangtze estuary, China. Environ. Geol. 41, 90-95 (2001).

24. Maskaoui, K., Zhou, J. L., Hong, H. S. \& Zhang, Z. L. Contamination by polycyclic aromatic hydrocarbons in the Jiulong River Estuary and Western Xiamen Sea, China. Environ. Pollut. 118, 109-122 (2002).

25. Tobiszewski, M. \& Namiesnik, J. PAH diagnostic ratios for the identification of pollution emission sources. Environ. Pollut. 162, 110-119 (2012).

26. Yunker, M. B. et al. PAHs in the Fraser River basin: a critical appraisal of $\mathrm{PAH}$ ratios as indicators of PAH source and composition. Org. Geochem. 33, 489-515 (2002)

27. Zhang, X. L. et al. Source diagnostics of polycyclic aromatic hydrocarbons based on species ratios: a multimedia approach. Environ. Sci. Technol. 39, 9109-9114 (2005).

28. Katsoyiannis, A., Sweetman, A. J. \& Jones, K. C. PAH molecular diagnostic ratios applied to atmospheric sources: a critical evaluation using two decades of source inventory and air concentration data from the UK. Environ. Sci. Technol. 45, 8897-8906 (2011).

29. Larsen, R. K. \& Baker, J. E. Source apportionment of polycyclic aromatic hydrocarbons in the urban atmosphere: a comparison of three methods. Environ. Sci. Technol. 37, 1873-1881 (2003).

30. Kavouras, I. G. et al. Source apportionment of urban particulate aliphatic and polynuclear aromatic hydrocarbons (PAHs) using multivariate methods. Environ. Sci. Technol. 35, 2288-2294 (2001).

31. Chen, Y. J. et al. Emission factors for carbonaceous particles and polycyclic aromatic hydrocarbons from residential coal combustion in China. Environ. Sci. Technol. 39, 1861-1867 (2005).

32. Dobbins, R. A., Fletcher, R. A., Benner, B. A. \& Hoeft, S. Polycyclic aromatic hydrocarbons in flames, in diesel fuels, an in diesel emissions. Combust. Flame 144, 773-781 (2006).

33. Shi, Z. et al. Dual mechanisms regulate ecosystem stability under decade-long warming and hay harvest. Nat. Commun. 7, 11973 (2016).

34. Trivedi, P. et al. Microbial regulation of the soil carbon cycle: evidence from gene-enzyme relationships. ISME J. 10, 2593-2604 (2016).

35. Grace, J. B. Structural Equation Modelling and Natural Systems (Cambridge Univ. Press, 2006)

\section{Acknowledgements}

This work was supported by the Basic Special Program of the Ministry of Science and Technology, China (2014FY210600), National Natural Science Foundation of China (41601525, 21677167 and 21976209), the Thousand Young Talents Program of China, Natural Science Foundation of Shandong Province (ZR2016DB07) and the Taishan Scholar Project Special Funding (ts20190962). We thank W. T. Shang, B. Hong and L. M. Liu for their help with data processing.

\section{Author contributions}

L.C., C.L. and D.L. designed the experiments. D.L. and D.W. collected the sediment samples and analysed sediment properties. C.L., M.L. and X.L. performed PAH analysis. M.L. collected anthropogenic and climatic parameters, analysed the data and wrote the original draft. G.Z. and G.J. provided experimental support and revision suggestions. All authors contributed extensively to conducting the experiments and revising the paper.

\section{Competing interests}

The authors declare no competing interests.

\section{Additional information}

Supplementary information is available for this paper at https://doi.org/10.1038/ s41893-020-0565-y.

Correspondence and requests for materials should be addressed to C.L., D.L. or L.C. Reprints and permissions information is available at www.nature.com/reprints. Publisher's note Springer Nature remains neutral with regard to jurisdictional claims in published maps and institutional affiliations.

(c) The Author(s), under exclusive licence to Springer Nature Limited 2020 


\section{natureresearch}

Corresponding author(s): Lingxin Chen; Chunyang Liao; Dongyan Liu

Last updated by author(s): Mar 2, 2020

\section{Reporting Summary}

Nature Research wishes to improve the reproducibility of the work that we publish. This form provides structure for consistency and transparency in reporting. For further information on Nature Research policies, see Authors \& Referees and the Editorial Policy Checklist.

\section{Statistics}

For all statistical analyses, confirm that the following items are present in the figure legend, table legend, main text, or Methods section.

n/a Confirmed

$\square$ \ The exact sample size $(n)$ for each experimental group/condition, given as a discrete number and unit of measurement

$\square$ \A statement on whether measurements were taken from distinct samples or whether the same sample was measured repeatedly

The statistical test(s) used AND whether they are one- or two-sided

Only common tests should be described solely by name; describe more complex techniques in the Methods section.

$\square$ \ A description of all covariates tested

$\square$ \A description of any assumptions or corrections, such as tests of normality and adjustment for multiple comparisons

$\square$ A full description of the statistical parameters including central tendency (e.g. means) or other basic estimates (e.g. regression coefficient)

$\triangle$ AND variation (e.g. standard deviation) or associated estimates of uncertainty (e.g. confidence intervals)

$\varnothing$ For null hypothesis testing, the test statistic (e.g. $F, t, r$ ) with confidence intervals, effect sizes, degrees of freedom and $P$ value noted

Give $P$ values as exact values whenever suitable.

Х $\square$ For Bayesian analysis, information on the choice of priors and Markov chain Monte Carlo settings

$\square$ \ For hierarchical and complex designs, identification of the appropriate level for tests and full reporting of outcomes

$\square$ ¿stimates of effect sizes (e.g. Cohen's $d$, Pearson's $r$ ), indicating how they were calculated

Our web collection on statistics for biologists contains articles on many of the points above.

\section{Software and code}

Policy information about availability of computer code

\section{Data collection No software was used.}

Data analysis No software was used.

For manuscripts utilizing custom algorithms or software that are central to the research but not yet described in published literature, software must be made available to editors/reviewers. We strongly encourage code deposition in a community repository (e.g. GitHub). See the Nature Research guidelines for submitting code \& software for further information.

\section{Data}

Policy information about availability of data

All manuscripts must include a data availability statement. This statement should provide the following information, where applicable:

- Accession codes, unique identifiers, or web links for publicly available datasets

- A list of figures that have associated raw data

- A description of any restrictions on data availability

The authors declare that the data supporting the findings of this study are summarized in the Supplementary Information, and any other data for analysis in the current study are available from the corresponding author upon request.

\section{Field-specific reporting}

Please select the one below that is the best fit for your research. If you are not sure, read the appropriate sections before making your selection. 


\section{Ecological, evolutionary \& environmental sciences study design}

All studies must disclose on these points even when the disclosure is negative.

Study description

To investigate how and to what extent anthropogenic factors influence PAHs distribution in the intertidal environment, the USEPA priority PAHs in sediments collected from 14 intertidal zones over $4500 \mathrm{~km}$ of Chinese coastlines, across all 11 coastal provinces and municipalities in China were measured, and a model disentangling the direct and indirect effects of various factors (anthropogenic factors, total organic carbon (TOC) content and climate factors) on profiles of PAHs in intertidal sediment was constructed.

Research sample

315 samples were obtained for dry and wet seasons. The sampling sites, spanning over all Chinese coastal provinces and municipalities, exhibit large variations in climate (temperature and rainfall) and anthropogenic factors (socio-economic parameters), which provides us a unique opportunity to investigate how diverse factors influence distribution of PAHs in intertidal zones.

Sampling strategy

14 intertidal zones were designed and chosen based on principles that they are distributed as evenly as possible and cover all coastal provinces and municipalities from about 41 degrees and 20 degrees north latitude spanning over $4500 \mathrm{~km}$ of Chinese coastlines. For each intertidal zone, based on the size of the intertidal zone, 3-5 transects perpendicular to the coast line were chosen and low, middle and high tides were usually set for each transect. The sampling sites were uniformly distributed in each intertidal zone to make them representative.

Data collection

Several institutes and universities were engaged in sampling and data collection using GPS and other in situ measurement devices. Concentrations of the 16 USEPA priority PAHs were measured by a Thermo Trace 1300 gas chromatography/TSQ 8000 EVO triple quadrupole mass detector system (GC-MS/MS) in SRM mode with an electron ionization (EI) ion source, using the two highest characteristic precursor ion/product ion transition pairs. Total organic carbon (TOC) content was measured with an elemental analyzer (Micro club Elementar, Germany). Particle size distribution of the sediment was analyzed using a laser diffraction particle size analyzer (Mastersizer 3000, UK). Anthropogenic parameters and climatic parameters (temperature and rainfall) were collected or estimated from governmental statistical yearbooks.

Timing and spatial scale During 2014-2015, surface sediments were taken from 14 intertidal zones across 11 Chinese provinces and municipalities in dry (September in 2014 to February in 2015) and wet seasons (July to September in 2015).

Data exclusions For statistical analysis, samples which were mainly sand were excluded to avoid the deviations caused by the measurement of total organic carbon (TOC) content. Statistical analysis was generally based on 261 samples.

Reproducibility

The pretreatment and analysis procedures including accelerated solvent extraction, cleaning up using alumina/silica column and GCMS/MS analysis were well established, extensively used and showed good reproducibilities. All attempts to repeat the experiment were successful.

Randomization When pretreating and analyzing the samples, a procedural blank, a spiked blank, a matrix spike and a duplicate were conducted for each batch of samples to make sure data reliable, and the samples for each batch were allocated randomly.

Blinding

Since the experimental object of this study was sediment, it seemed blinding was not relevant to this study.

Did the study involve field work? \Yes $\square$ No

\section{Field work, collection and transport}

Field conditions

Location

Access and import/export
Surface sediments were sampled from 14 intertidal zones in both dry and wet seasons. The temperature and rainfall were in the range of $6.9-29^{\circ} \mathrm{C}$ and $14.2-424.5 \mathrm{~mm}$, respectively, with detailed information listed in Supplementary Table 2.

The sampling was conducted by taking surface sediment from 14 intertidal zones over $4500 \mathrm{~km}$ of Chinese coastlines, with latitude ranging from 19.998 to 40.610 and longitude ranging from 109.747 to 122.190 , and detailed information can be found in the manuscript.

The sampling was conducted in compliance with the local and national laws, with introduction letter from the institute and permissions of the local Management Agencies.

This study sampled surface sediment during the low tide period. On the same day, when the high tide passed, the sites will soon get back to their original. Almost no disturbance was caused by this study.

\section{Reporting for specific materials, systems and methods}

We require information from authors about some types of materials, experimental systems and methods used in many studies. Here, indicate whether each material, system or method listed is relevant to your study. If you are not sure if a list item applies to your research, read the appropriate section before selecting a response. 
Materials \& experimental systems Methods

\begin{tabular}{|c|c|c|c|}
\hline $\mathrm{n} / \mathrm{a}$ & Involved in the study & $\mathrm{n} / \mathrm{a}$ & Involved in the study \\
\hline Х & Antibodies & Х & ChIP-seq \\
\hline 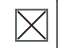 & Eukaryotic cell lines & Х & Flow cytometry \\
\hline Х & $\square$ Palaeontology & $\bigotimes$ & $\square$ MRI-based neuroimaging \\
\hline$\bigotimes$ & $\square$ Animals and other organisms & & \\
\hline Х & $\square$ Human research participants & & \\
\hline Х & Clinical data & & \\
\hline
\end{tabular}

